\title{
Probability Distribution and Fuzzy System Based on Triple I Algorithm
}

\author{
Hongwei $\mathrm{Fu}^{1, \mathrm{a}}$,Liang Yang ${ }^{1, \mathrm{a}}$, Sichong Qian ${ }^{1, \mathrm{a}}$ \\ ${ }^{1}$ Department of Basic Education, Military Economy Academy, Wuhan, 430035, China. \\ ahwfu123@163.com
}

Keywords: triple I algorithm, generalized center of gravity fuzzy system, center of gravity fuzzy system, probability distribution, universal approximation.

Abstract: In the paper, probability distribution and fuzzy system based on the triple I algorithm and modified Reichenbach operator are discussed. Firstly, the joint probability density functions of two-dimensional random variables, the marginal probability density functions and numerical characteristics are obtained by using the center of gravity defuzzifier method. Secondly, the generalized center of gravity fuzzy systems and the center of gravity fuzzy systems based on singleton fuzzier method and non-singleton fuzzier method are obtained respectively. Meanwhile, it is pointed out that the center of gravity fuzzy systems are regression functions in the sense of probability theory. Finally, we prove the fuzzy systems not only have the universal approximation but also possess the first-order approximation accuracy.

\section{Introduction}

Since the fuzzy set theory was introduced in $1965^{[1 \sim 3]}$, fuzzy control theories have achieved a series of remarkable success ${ }^{[4]}$. The construction of fuzzy system is being paid more and more attention by scholars. It is well known that fuzzy system consists of four principle components: fuzzifier, fuzzy rules, fuzzy inference engine and defuzzifier.

The fuzzy system is usually constructed by singleton fuzzifier, CRI method and center average defuzzifier. But for some implication operators, the fuzzy system based on the singleton fuzzifier may not have the universal approximation ${ }^{[5]}$. As we know, the triple I algorithm adopts implication operator for three times while the CRI algorithm only adopts once ${ }^{[6]}$. From a certain point of view, the triple I algorithm is strict and its calculating result is similar to the result of CRI, even more perfect sometimes ${ }^{[7]}$. Fuzzy system based on the center of gravity defuzzifier was proved to be some interpolation function ${ }^{[8]}$. Then, Probability distribution based on the center of gravity defuzzifier has been established in the paper ${ }^{[9]}$.

Based on the above work, we get the corresponding probability density functions for the fuzzy implication operator through a set of input - output data. Compared with the numerical characteristics in the paper ${ }^{[10]}$, we get the conclusion that using different implications we can get varying probability density functions, but their corresponding random variables have the same mathematical expectations, the similar variance and covariance. Finally, we give the mathematical expressions of the fuzzy systems constructed based on the generalized center of gravity defuzzifier and the center of gravity defuzzifier and point out that the center of gravity fuzzy systems is a regression function in the sense of probability theory and they qualify for the first-order approximation accuracy.

The paper is organized as follows. In section 2, based on the singleton fuzzifier and the non-singleton fuzzifier, we discuss the probability distributions of the center of gravity fuzzy systems respectively. In section 3, we model the fuzzy systems based on the generalized center of 
gravity defuzzifier and the center of gravity defuzzifier respectively. Meanwhile, we study the universal approximations of the center of gravity fuzzy systems. In section 4, we draw the conclusion.

Then we introduce several concepts which are used in this paper.

\section{Definition 1.1. Fuzzifier:}

(1)Singleton fuzzifier: change the input variable into the fuzzy set, that is

$A^{*}\left(x^{\prime}\right)=\left\{\begin{array}{ll}1 & , x^{\prime}=x \\ 0 & , x^{\prime} \neq x\end{array}\right.$.

(2)Non-singleton fuzzifier: change the input variable into the fuzzy set, that is

$$
A^{*}\left(x^{\prime}\right)=\left\{\begin{array}{ll}
A(x) & , x^{\prime}=x \\
0 & , x^{\prime} \neq x
\end{array} .\right.
$$

Definition 1.2. Triple implication algorithm (triple I algorithm): let $A, A^{*} \in F(X), B \in F(Y)$, then we have

$$
(A(x) \rightarrow B(y)) \rightarrow\left(A^{*}(x) \rightarrow B^{*}(y)\right) .
$$

In formula (3), $B^{*}$ is the minimum fuzzy set which can make (3) have the maximum.

Especially, $B^{*}$ is the minimum fuzzy set in ${ }_{F(Y)}$, which can make (3) equal to 1 constantly, while the implication operator satisfies the conditions" $a \leq b, a \rightarrow b=1$ ".

Definition 1.3. Generalized center of gravity fuzzy system can be expressed as following,

$$
H(x)=\frac{\sum_{i=1}^{n} y_{i} B^{*}\left(y_{i}\right) \Delta y_{i}}{\sum_{i=1}^{n} B^{*}\left(y_{i}\right) \Delta y_{i}}
$$

Definition 1.4. Center of gravity fuzzy system can be expressed as following,

$$
\bar{S}(x)=\frac{\int_{-\infty}^{+\infty} y B^{*}(y) \mathrm{d} y}{\int_{-\infty}^{+\infty} B^{*}(y) \mathrm{d} y} .
$$

\section{Probability Distributions of the Center of Gravity Fuzzy Systems}

Suppose $\left\{\left(x_{i}, y_{i}\right)\right\}(1 \leq i \leq n)$ to be a set of input-output data, and

$$
a=x_{1}<x_{2}<\mathrm{L}<x_{n}=b, c=y_{1}<y_{2}<\mathrm{L}<y_{n}=d \text { or } a=x_{1}<x_{2}<\mathrm{L}<x_{n}=b, c=y_{n}<y_{n-1}<\mathrm{L}<y_{1}=d
$$

Then, we can construct the triangle wave with duality by the known data

$$
\begin{aligned}
& \quad A_{i}(x), B_{i}(y)(i=1,2, \mathrm{~L}, n) \text { and } A_{i}\left(x_{i}\right)=1, B_{i}\left(y_{i}\right)=1(i=1,2, \ldots \ldots, n) . \\
& \text { When } x \in\left[x_{i}, x_{i+1}\right], A_{i}(x)+A_{i+1}(x)=1, A_{j}(x)=0(j \neq i, i+1), \\
& \text { When } y \in\left[y_{i}, y_{i+1}\right], B_{i}(y)+B_{i+1}(y)=1, B_{j}(y)=0(j \neq i, i+1) . \\
& \text { Then we can have fuzzy inference rules: } \\
& \text { If } x \text { is } A_{i} \text {, then } y \text { is } B_{i}(i=1,2, \mathrm{~L}, n) .
\end{aligned}
$$


Let $\theta(a, b)=\left\{\begin{array}{l}1 \quad, a \leq b \\ 1-a+a b, a>b\end{array}\right.$ is the fuzzy implication operator, we can have the fuzzy inference relation based on (6): $R(x, y)=\stackrel{n}{\vee} \vee_{i=1} \theta\left(A_{i}(x), B_{i}(y)\right)$.

Then let

$H(2, n, \theta, \vee) \quad \int_{-\infty}^{+\infty} \int_{-\infty}^{+\infty} R(x, y) \mathrm{d} y \mathrm{~d} x$.

$$
f(x, y) \frac{R(x, y)}{H(2, n, \theta, \vee)} \text {. }
$$

We can have $(a) f(x, y)>0 ; \quad(b) \int_{-\infty}^{+\infty} \int_{-\infty}^{+\infty} f(x, y) \mathrm{d} y \mathrm{~d} x=1$. Therefore, $f(x, y)$ can be regarded as the joint probability density of the random vector $(\xi, \eta)$.

Since the data in this paper is monotonous, the fuzzy inference relationship is modified as:

$R(x, y)=\left\{\begin{array}{cc}\theta\left(A_{i}(x), B_{i}(y)\right) \vee \theta\left(A_{i+1}(x), B_{i+1}(y)\right),(x, y) \in I_{i} \times J_{i} \\ 0, & \text { else }\end{array}\right.$

Where, $I_{i}=\left[x_{i}, x_{i+1}\right], J_{i}=\left[y_{i}, y_{i+1}\right]$.

Theorem $2.1 B^{*}(y)$ based on the triple I algorithm can be expressed as

$B^{*}(y)=\underset{x \in E_{y}}{\vee}\left[A^{*}(x) \wedge \frac{A(x) \cdot B(y)-A(x)+A^{*}(x)}{A^{*}(x)}\right]$.

Where, $\quad E_{y}=\left\{x \in X \mid R(A(x), B(y))+A^{*}(x) \geq 1\right.$, and $\left.A^{*}(x)>0\right\}$.

Proof: Let $H(x, y)=(A(x) \rightarrow B(y)) \rightarrow\left(A^{*}(x) \rightarrow B^{*}(y)\right)$, we can have

$H(x, y)=R(A(x), B(y)) \rightarrow R\left(A^{*}(x), B^{*}(y)\right)$

$=\left\{\begin{array}{lr}1 & , R(A(x), B(y)) \leq R\left(A^{*}(x), B^{*}(y)\right) \\ 1-R(A(x), B(y))+R(A(x), B(y)) \cdot R\left(A^{*}(x), B^{*}(y)\right), & R(A(x), B(y))>R\left(A^{*}(x), B^{*}(y)\right)\end{array}\right.$

Let $R(A(x), B(y)) \leq R\left(A^{*}(x), B^{*}(y)\right)=\left\{\begin{array}{ll}1 & , A^{*}(x) \leq B^{*}(y) \\ 1-A^{*}(x)+A^{*}(x) \cdot B^{*}(y), A^{*}(x)>B^{*}(y)\end{array}\right.$,

we can make the maxmum of $H(x, y)$ equal to 1 .

When $A^{*}(x) \leq B^{*}(y)$, the above formula holds for the fixed $(x, y)$.

When $A^{*}(x)>B^{*}(y),{ }^{1-A(x)}+A(x) \cdot B(y) \leq 1-A^{*}(x)+A^{*}(x) \cdot B^{*}(y)$, namely,

$B^{*}(y) \geq \frac{R(A(x), B(y))+A^{*}(x)-1}{A^{*}(x)}=\frac{A(x) \cdot B(y)-A(x)+A^{*}(x)}{A^{*}(x)}$.

Therefore,

$$
B^{*}(y) \geq \underset{x \in E_{y}}{\vee}\left[A^{*}(x) \wedge \frac{R(A(x), B(y))+A^{*}(x)-1}{A^{*}(x)}\right]=B \quad(y)
$$

At the same time,

$$
\forall x \in E_{y}, B \quad(y) \geq A^{*}(x) \wedge \frac{R(A(x), B(y))+A^{*}(x)-1}{A^{*}(x)}
$$

So, we have

$$
B \quad(y) \geq A^{*}(x) \text { or } B \quad(y) \geq \frac{R(A(x), B(y))+A^{*}(x)-1}{A^{*}(x)}
$$

and $H(x, y)=(A(x) \rightarrow B(y)) \rightarrow\left(A^{*}(x) \rightarrow B^{\Delta}(x)\right)=1$. 
Then, we can get the minimum fuzzy set

$$
B^{*}(y)=\underset{x \in E_{y}}{\vee}\left[A^{*}(x) \wedge \frac{A(x) \cdot B(y)-A(x)+A^{*}(x)}{A^{*}(x)}\right] .
$$

On the basis of the above work, using the singleton fuzzifier, we can obtain

$$
B^{*}(y)=1-A(x)+A(x) B(y) .
$$

Using the non-singleton fuzzifier, we can have $B^{*}(y)=A(x) \wedge B(y)$.

Theorem 2.2 Let $L=\frac{11}{12} \sum_{i=1}^{n-1}\left(x_{i+1}-x_{i}\right)\left(y_{i+1}-y_{i}\right)$, using the singleton fuzzifier and triple I algorithm, the joint density function of the fuzzy system can be expressed as following,

$$
f(x, y)=\frac{q(x, y)}{H(2, n, \theta, \vee)}=\left\{\begin{array}{cc}
\frac{1}{L}\left(1-A_{i}(x) B_{i+1}(y)\right), & \exists i,(x, y) \in D_{i} \\
\frac{1}{L}\left(1-A_{i+1}(x) B_{i}(y)\right), & \exists i,(x, y) \in E_{i} \\
0, & \text { else }
\end{array} .\right.
$$

Where,

$$
\begin{aligned}
& I_{i}=\left[x_{i}, x_{i+1}\right], J_{i}=\left[y_{i}, y_{i+1}\right], D_{i}=\left\{(x, y) \leq I_{i} \times J_{i} \mid y \in A_{i+1}(x) y_{i+1}+A_{i}(x) y_{i}\right\}, \\
& E_{i}=\left\{(x, y) \in I_{i} \times J_{i} \mid y>A_{i+1}(x) y_{i+1}+A_{i}(x) y_{i}\right\}
\end{aligned}
$$

Proof: By (10), we can have

$$
\begin{aligned}
R(x, y) & =\left[1-A_{i}(x)+A_{i}(x) B_{i}(y)\right] \vee\left[1-A_{i+1}(x)+A_{i+1}(x) B_{i+1}(y)\right] \\
& =\left[1-A_{i}(x) B_{i+1}(y)\right] \vee\left[1-A_{i+1}(x) B_{i}(y)\right]
\end{aligned}
$$

By (7), we can have

$$
\begin{aligned}
H(2, n, \theta, \vee) & =\sum_{i=1}^{n-1} \int_{x_{i}}^{x_{i+1}} \int_{y_{i}}^{y_{i+1}} R(x, y) \mathrm{d} y \mathrm{~d} x \\
& =\sum_{i=1}^{n-1} \int_{x_{i}}^{x_{i+1}} \int_{y_{i}}^{y_{i+1}}\left(1-A_{i}(x) B_{i+1}(y)\right) \vee\left(1-A_{i+1}(x) B_{i}(y)\right) \mathrm{d} y \mathrm{~d} x \\
& =\sum_{i=1}^{n-1} \int_{x_{i}}^{x_{i+1}}\left(y_{i+1}-y_{i}\right)\left(1-\frac{1}{2} A_{i}(x) A_{i+1}(x)\right) d x \\
& =\frac{11}{12} \sum_{i=1}^{n-1}\left(x_{i+1}-x_{i}\right)\left(y_{i+1}-y_{i}\right) \quad L
\end{aligned}
$$

Then, we can obtain the joint density function:

$$
f(x, y)=\frac{q(x, y)}{H(2, n, \theta, \vee)}=\left\{\begin{array}{cc}
\frac{1}{L}\left(1-A_{i}(x) B_{i+1}(y)\right), & \exists i,(x, y) \in D_{i} \\
\frac{1}{L}\left(1-A_{i+1}(x) B_{i}(y)\right), & \exists i,(x, y) \in E_{i} \\
0, & \text { els e }
\end{array}\right.
$$

Theorem 2.3 Based on (12), the marginal density functions and the numerical characters can be expressed as 
(1) $f_{\zeta}(x)=\frac{1}{2 L}\left(y_{i+1}-y_{i}\right)\left[2-A_{i}(x) A_{i+1}(x)\right] \quad\left(x \in\left[x_{i}, x_{i+1}\right], i=1,2, \mathrm{~L}, n\right)$

$f_{\eta}(y)=\frac{1}{2 L}\left(x_{i+1}-x_{i}\right)\left[1-B_{i}(y) B_{i+1}(y)\right] \quad\left(y \in\left[y_{i}, y_{i+1}\right], i=1,2, \mathrm{~L}, n\right)$

(2) $E(X)=\sum_{i=1}^{n-1} w_{i} \bar{x}_{i}, \quad E(Y)=\sum_{i=1}^{n-1} w_{i} \bar{y}_{i}$.

(3) $D(X) \approx \sum_{i=1}^{n-1} w_{i} x_{i} x_{i+1}-\left(\sum_{i=1}^{n-1} w_{i} \bar{x}_{i}\right)^{2}, D(Y) \approx \sum_{i=1}^{n-1} w_{i} y_{i} y_{i+1}-\left(\sum_{i=1}^{n-1} w_{i} \bar{y}_{i}\right)^{2}$.

(4) $\operatorname{Cov}(X, Y) \approx \sum_{i=1}^{n-1} w_{i} \bar{z}_{i}-\left(\sum_{i=1}^{n-1} w_{i} \bar{x}_{i}\right)\left(\sum_{i=1}^{n-1} w_{i} \bar{y}_{i}\right)$.

Where,

$$
\begin{aligned}
& w_{i}=\frac{\left(y_{i+1}-y_{i}\right)\left(x_{i+1}-x_{i}\right)}{\sum_{k=1}^{n-1}\left(y_{k+1}-y_{k}\right)\left(x_{k+1}-x_{k}\right)}, \bar{x}_{i}=\frac{1}{2}\left(x_{i}+x_{i+1}\right), \bar{y}_{i}=\frac{1}{2}\left(y_{i}+y_{i+1}\right), \\
& \bar{z}_{i}=\frac{1}{2}\left(x_{i} y_{i}+x_{i+1} y_{i+1}\right)(i=1,2, \mathrm{~L}, n-1) .
\end{aligned}
$$

Proof: (1) When $x \in I_{i}$, let $y_{i}^{*}=A_{i}(x) y_{i}+A_{i+1}(x) y_{i+1}$, we can have

$$
\begin{aligned}
f_{\xi}(x) & =\int_{-\infty}^{+\infty} f(x, y) d y=\int_{y_{i}}^{y_{i+1}} f(x, y) d y \\
& =\frac{1}{L}\left[\int_{y_{i}}^{y_{i}^{*}}\left(1-A_{i}(x) B_{i+1}(y)\right) \mathrm{d} y+\int_{y_{i}^{*}}^{y_{i+1}}\left(1-A_{i+1}(x) B_{i}(y)\right) \mathrm{d} y\right] \\
& =\frac{y_{i+1}-y_{i}}{2 L}\left[2-A_{i}(x) A_{i+1}(x)\right]
\end{aligned}
$$

Similarly, we can get

$$
f_{\eta}(y)=\frac{\left(x_{i+1}-x_{i}\right)}{2 L}\left[1-B_{i}(y) B_{i+1}(y)\right]
$$

$$
\begin{aligned}
& \begin{array}{l}
E(X)=\int_{-\infty}^{+\infty} x f_{\zeta}(x) d x=\sum_{i=1}^{n-1} \int_{x_{i}}^{x_{i+1}} x f_{\zeta}(x) \mathrm{d} x=\sum_{i=1}^{n-1} \frac{1}{2 L}\left(y_{i+1}-y_{i}\right) \int_{x_{i}}^{x_{i+1}} x\left(2-A_{i}(x) A_{i+1}(x)\right) \mathrm{d} x \\
=\sum_{i=1}^{n-1} \frac{11}{12 L}\left(y_{i+1}-y_{i}\right)\left(x_{i+1}-x_{i}\right) \bar{x}_{i}=\sum_{i=1}^{n-1} w_{i} \bar{x}_{i}
\end{array} \\
& \text { Similarly, } E(Y)=\sum_{i=1}^{n-1} w_{i} \bar{y}_{i} .
\end{aligned}
$$

(3)

$$
\begin{aligned}
E\left(X^{2}\right) & =\int_{-\infty}^{+\infty} x^{2} f_{\zeta}(x) d x=\sum_{i=1}^{n-1} \int_{x_{i}}^{x_{i+1}} x^{2} f_{\zeta}(x) \mathrm{d} x=\sum_{i=1}^{n-1} \frac{y_{i+1}-y_{i}}{2 L} \int_{x_{i}}^{x_{i+1}} x^{2}\left(2-A_{i}(x) A_{i+1}(x)\right) \mathrm{d} x \\
& =\sum_{i=1}^{n-1} \frac{\left(y_{i+1}-y_{i}\right)\left(x_{i+1}-x_{i}\right)}{2 L}\left[\frac{11}{6} x_{i} x_{i+1}+\frac{37}{60}\left(x_{i+1}-x_{i}\right)^{2}\right] \\
& =\sum_{i=1}^{n-1} w_{i} x_{i} x_{i+1}+\frac{37}{110} \sum_{i=1}^{n-1} w_{i}\left(x_{i+1}-x_{i}\right)^{2}
\end{aligned}
$$

When $n \rightarrow \infty, \max _{1 \leq i \leq n-1}\left(x_{i+1}-x_{i}\right)^{2} \rightarrow 0$. Thus, $E\left(X^{2}\right) \approx \sum_{i=1}^{n-1} w_{i} x_{i} x_{i+1}$, 
$D(X)=E\left(X^{2}\right)-(E(X))^{2} \approx \sum_{i=1}^{n-1} w_{i} x_{i} x_{i+1}-\left(\sum_{i=1}^{n-1} w_{i} \bar{x}_{i}\right)^{2}$.

Similarly, $D(Y) \approx \sum_{i=1}^{n-1} w_{i} y_{i} y_{i+1}-\left(\sum_{i=1}^{n-1} w_{i} \bar{y}_{i}\right)^{2}$.

(4)

$$
\begin{aligned}
E(X Y) & =\int_{-\infty}^{+\infty} \int_{-\infty}^{+\infty} x y f(x, y) \mathrm{d} y \mathrm{~d} x=\sum_{i=1}^{n-1} \iint_{I_{i} \times J_{i}} x y f(x, y) \mathrm{d} y \mathrm{~d} x \\
& =\sum_{i=1}^{n-1}\left[\iint_{D_{i}} x y \frac{1}{L}\left(1-A_{i}(x) B_{i+1}(y)\right) \mathrm{d} y \mathrm{~d} x+\iint_{E_{i}} x y \frac{1}{L}\left(1-A_{i+1}(x) B_{i}(y)\right) \mathrm{d} y \mathrm{~d} x\right] \\
& =\frac{1}{L} \sum_{i=1}^{n-1}\left(\frac{11}{24}\left(x_{i} y_{i}+x_{i+1} y_{i+1}\right)\right)\left(y_{i+1}-y_{i}\right)\left(x_{i+1}-x_{i}\right) \\
& \approx \sum_{i=1}^{n-1} \omega_{i}\left[\frac{1}{2}\left(x_{i} y_{i}+x_{i+1} y_{i+1}\right)\right]=\sum_{i=1}^{n-1} \omega_{i} \bar{z}_{i} \\
\text { Thus, } \quad & \operatorname{Cov}(X, Y)=E(X Y)-E(X) E(Y) \approx \sum_{i=1}^{n-1} \omega_{i} \bar{z}_{i}-\left(\sum_{i=1}^{n-1} \omega_{i} \bar{x}_{i}\right)\left(\sum_{i=1}^{n-1} \omega_{i} \bar{y}_{i}\right) .
\end{aligned}
$$

Theorem 2.4 Let $L^{\prime}=\frac{1}{2} \sum_{i=1}^{n-1}\left(x_{i+1}-x_{i}\right)\left(y_{i+1}-y_{i}\right)$ and the definitions of $\bar{x}_{i}, \bar{y}_{i}, \bar{z}_{i}$ are same as those in the theorem 2.3, using the non-singleton fuzzifier and triple I algorithm, we have the probability distribution of the fuzzy system.

the joint density function:

$$
f(x, y)=\left\{\begin{array}{ll}
\frac{1}{L^{\prime}}\left(A_{i}(x) \wedge B_{i}(y)\right), & \exists i,(x, y) \in D_{i}^{\prime} \\
\frac{1}{L^{\prime}}\left(A_{i+1}(x) \wedge B_{i+1}(y)\right), & \exists i,(x, y) \in E_{i}^{\prime} \\
0, & \text { else }
\end{array}\right\} .
$$

Where, $\quad D_{i}^{\prime}=\left\{(x, y) \in I_{i} \times J_{i} \mid y \leq A_{i+1}(x) y_{i}+A_{i}(x) y_{i+1}\right\}$

$$
E_{i}^{\prime}=\left\{(x, y) \in I_{i} \times J_{i} \mid y>A_{i+1}(x) y_{i}+A_{i}(x) y_{i+1}\right\}
$$

the marginal density function, mathematical expectation, variance and covariance:

(1) $f_{\xi}(x)=\frac{y_{i+1}-y_{i}}{2 L^{\prime}}, x \in I_{i} ; \quad f_{\eta}(y)=\frac{x_{i+1}-x_{i}}{2 L^{\prime}}, y \in J_{i}$.

(2) $E(X)=\sum_{i=1}^{n-1} \omega_{i} \bar{x}_{i}, E(Y)=\sum_{i=1}^{n-1} \omega_{i} \bar{y}_{i}$.

$D(X) \approx \sum_{i=1}^{n-1} \omega_{i} x_{i} x_{i+1}-\left(\sum_{i=1}^{n-1} \omega_{i} \bar{x}_{i}\right)^{2}, \quad D(Y) \approx \sum_{i=1}^{n-1} \omega_{i} y_{i} y_{i+1}-\left(\sum_{i=1}^{n-1} \omega_{i} \bar{y}_{i}\right)^{2}$.

(4) $\operatorname{Cov}(X, Y) \approx \sum_{i=1}^{n-1} \omega_{i} \bar{z}_{i}-\left(\sum_{i=1}^{n-1} \omega_{i} \bar{x}_{i}\right)\left(\sum_{i=1}^{n-1} \omega_{i} \bar{y}_{i}\right)$.

Proof: Omit.

Remark 2.1 When we make equidistant partition on $X, Y$ and let $h=(b-a) /(n-1), \quad k=(d-c) /(n-1)$, we can have 
(1) $E(X)=\frac{a+b}{2}, \quad E(Y)=\frac{c+d}{2}$;

(2) $\lim _{n \rightarrow \infty} D(X)=\frac{(b-a)^{2}}{12}, \quad \lim _{n \rightarrow \infty} D(Y)=\frac{(d-c)^{2}}{12}$;

(3) $\lim _{n \rightarrow \infty} \operatorname{Cov}(X, Y)=\frac{(b-a)(d-c)}{12}$;

Remark 2.2 From the theorem 2.3 and 2.4, we know that the fuzzy systems based on different methods have the same numerical characters. Compared with the paper ${ }^{[11]}$, we can say that varying functions based on different fuzzy implication operators can be obtained while their corresponding random variables have the same mathematical expectations, the similar variance and covariance.

\section{Fuzzy Systems and Universal Approximation}

Theorem 3.1 The generalized center of gravity fuzzy system based on the singleton fuzzifier and triple I algorithm can be expressed as

$$
H(x)=\frac{y_{i} \Delta y_{i}+y_{i+1} \Delta y_{i+1}}{\Delta y_{i}+\Delta y_{i+1}} \text {. }
$$

Proof: When $x \in\left[x_{i}, x_{i+1}\right]$, by (10) we have

$$
\begin{aligned}
& \begin{aligned}
\begin{aligned}
B^{*}(y) & =R(x, y)=1-A(x)+A(x) \cdot B(y) \\
& =\left(1-A_{i}(x)+A_{i}(x) \cdot B_{i}(y)\right) \vee\left(1-A_{i+1}(x)+A_{i+1}(x) \cdot B_{i+1}(y)\right) \\
= & {\left[1-A_{i}(x) B_{i+1}(y)\right] \vee\left[1-A_{i+1}(x) B_{i}(y)\right] }
\end{aligned} \\
\text { Then, } \quad B^{*}\left(y_{j}\right)=\left\{\begin{array}{l}
1, j=i, i+1 \\
0, j \neq i, i+1
\end{array}\right. \\
\text { By (4), we can have } H(x)=\frac{y_{i} \Delta y_{i}+y_{i+1} \Delta y_{i+1}}{\Delta y_{i}+\Delta y_{i+1}} .
\end{aligned}
\end{aligned}
$$

Theorem 3.2 The generalized center of gravity fuzzy system based on the non-singleton fuzzifier and triple I algorithm can be expressed as

$$
H(x)=\frac{A_{i}(x) y_{i} \Delta y_{i}+A_{i+1}(x) y_{i+1} \Delta y_{i+1}}{A_{i}(x) \Delta y_{i}+A_{i+1}(x) \Delta y_{i+1}} .
$$

Proof: When $x \in\left[x_{i}, x_{i+1}\right]$, by (11) we have

$$
B^{*}(y)=A(x) \wedge B(y)=\left[A_{i}(x) \wedge B_{i}(y)\right] \vee\left[A_{i+1}(x) \wedge B_{i+1}(y)\right]
$$

Then,

$$
B^{*}\left(y_{j}\right)=\underset{i=1}{\vee} B_{i}^{*}\left(y_{j}\right)=\left\{\begin{array}{l}
A_{i}(x) \quad j=i \\
A_{i+1}(x) \quad j=i+1 \\
0 \quad j \neq i, i+1
\end{array}\right.
$$

By (4), we can have

$$
H(x)=\frac{A_{i}(x) y_{i} \Delta y_{i}+A_{i+1}(x) y_{i+1} \Delta y_{i+1}}{A_{i}(x) \Delta y_{i}+A_{i+1}(x) \Delta y_{i+1}}
$$

Assume $f(x, y)$ is the joint density function in this paper and $\bar{s}(x)$ is the fuzzy system, by the theorem 1.4 , theorem 2.2 , theorem 2.4 , we can have

$$
E(\eta \mid \xi=x)=\frac{\int_{-\infty}^{+\infty} y f(x, y) \mathrm{d} y}{\int_{-\infty}^{+\infty} f(x, y) \mathrm{d} y}=\frac{\int_{c}^{d} y R(x, y) \mathrm{d} y}{\int_{c}^{d} R(x, y) \mathrm{d} y}=\frac{\int_{c}^{d} y B^{*}(y) \mathrm{d} y}{\int_{c}^{d} B^{*}(y) \mathrm{d} y}=\bar{S}(x)
$$

Thus, $\bar{s}(x)$ is a regression function of $y$ about $x$. Namely, the center of gravity fuzzy system is the regression function in the probability theory. So, we have the following theorems. 
Theorem 3.3 (1) The center of gravity fuzzy system based on the singleton fuzzifier and triple I algorithm can be expressed as

$$
\overline{S_{1}}(x)=A_{i}^{*}(x) y_{i}+A_{i+1}^{*}(x) y_{i+1} .
$$

Where,

$$
A_{i+1}^{*}(x)=\left(\frac{1+\frac{1}{3} A_{i+1}(x)-\frac{2}{3} A_{i+1}(x) A_{i}(x)}{2-A_{i+1}(x) A_{i}(x)}\right), A_{i}^{*}(x)=\frac{1-\frac{1}{3} A_{i+1}(x) A_{i}(x)-\frac{1}{3} A_{i+1}(x)}{2-A_{i+1}(x) A_{i}(x)} .
$$

(2) The center of gravity fuzzy system based on the non-singleton fuzzifier and triple I algorithm can be expressed as

$$
\bar{S}_{2}(x)=A_{i}(x) y_{i}+A_{i+1}(x) y_{i+1} .
$$

Where,

$A_{i}(x)=\frac{1}{3}+A_{i}^{2}(x)-\frac{2}{3} A_{i}^{3}(x), A_{i+1}(x)=1-A_{i}(x)=\frac{1}{3}+A_{i+1}^{2}(x)-\frac{2}{3} A_{i+1}^{3}(x)$

Proof: (1) When ${ }^{x \in\left[x_{i}, x_{i+1}\right]}$, let $y_{i}^{*}=A_{i}(x) y_{i}+A_{i+1}(x) y_{i+1}$, we can have

$$
\begin{aligned}
& \int_{-\infty}^{+\infty} y f(x, y) \mathrm{d} y=\frac{1}{L} \int_{y_{i}}^{y_{i}^{*}} y\left(1-A_{i}(x) B_{i+1}(y)\right) \mathrm{d} y+\frac{1}{L} \int_{y_{i}^{*}}^{y_{i+1}} y\left(1-A_{i+1}(x) B_{i}(y)\right) \mathrm{d} y \\
& =\frac{y_{i+1}-y_{i}}{L}\left[y_{i}\left(\frac{1}{2}-\frac{1}{6} A_{i}(x) A_{i+1}(x)-\frac{1}{6} A_{i+1}(x)\right)+y_{i+1}\left(\frac{1}{2}-\frac{1}{3} A_{i}(x) A_{i+1}(x)+\frac{1}{6} A_{i+1}(x)\right)\right] \\
& \int_{-\infty}^{+\infty} f(x, y) \mathrm{d} y=f_{\zeta}(x)=\frac{y_{i+1}-y_{i}}{2 L}\left(2-A_{i}(x) A_{i+1}(x)\right)
\end{aligned}
$$

Then,

$$
\begin{aligned}
& \bar{S}_{1}(x)=\frac{\int_{-\infty}^{+\infty} y f(x, y) \mathrm{d} y}{\int_{-\infty}^{+\infty} f(x, y) \mathrm{d} y} \\
& =\frac{\frac{y_{i+1}-y_{i}}{L}\left[y_{i}\left(\frac{1}{2}-\frac{1}{6} A_{i}(x) A_{i+1}(x)-\frac{1}{6} A_{i+1}(x)\right)+y_{i+1}\left(\frac{1}{2}-\frac{1}{3} A_{i}(x) A_{i+1}(x)+\frac{1}{6} A_{i+1}(x)\right)\right]}{\frac{1}{2 L}\left(y_{i+1}-y_{i}\right)\left(2-A_{i+1}(x) A_{i}(x)\right)} \\
& =\left(\frac{1-\frac{1}{3} A_{i+1}(x) A_{i}(x)-\frac{1}{3} A_{i+1}(x)}{2-A_{i+1}(x) A_{i}(x)}\right) \cdot y_{i}+\left(\frac{1+\frac{1}{3} A_{i+1}(x)-\frac{2}{3} A_{i+1}(x) A_{i}(x)}{2-A_{i+1}(x) A_{i}(x)}\right) \cdot y_{i+1}
\end{aligned}
$$

We can have $\bar{S}_{1}(x)=A_{i}^{*}(x) y_{i}+A_{i+1}^{*}(x) y_{i+1}$.

Similarly, we can proof (17).

Theorem 3.4 Assume $s(x)$ is the original system and ${ }^{s\left(x_{i}\right)=y_{i}}$. Then we have

$$
\begin{aligned}
& \left\|\overline{S_{1}}-s\right\|_{\infty} \leq \frac{1}{8}\left\|s^{\prime}\right\|_{\infty} h^{2}+\frac{1}{2}\left\|s^{\prime}\right\|_{\infty} h . \\
& \left\|\overline{S_{2}}-s\right\|_{\infty} \leq \frac{1}{8}\left\|s^{\prime}\right\|_{\infty} h^{2}+\frac{1}{3}\left\|s^{\prime}\right\|_{\infty} h . \\
& \text { Where, } h=\max _{1 \leq i \leq n-1} \Delta x_{i},\|s\|_{\infty}=\max _{x \in[a, b]}|s(x)| .
\end{aligned}
$$


Proof: (1) Let $F_{1}(x)=A_{i}(x) \cdot y_{i}+A_{i+1}(x) \cdot y_{i+1}\left(x \in\left[x_{i}, x_{i+1}\right]\right)$, then

$\left\|\overline{S_{1}}-s\right\|_{\infty} \leq\left\|\overline{S_{1}}-F_{1}\right\|_{\infty}+\left\|s-F_{1}\right\|_{\infty}$

Based on paper [12], we can know

$$
\left\|s-F_{1}\right\| \leq \frac{1}{8}\left\|s^{\prime \prime}\right\|_{\infty} h^{2}
$$

Since

$$
\begin{aligned}
& \left|\overline{S_{1}}(x)-F_{1}(x)\right|=\left|A_{i}^{*}(x) y_{i}+A_{i+1}^{*}(x) y_{i+1}-A_{i}(x) \cdot y_{i}-A_{i+1}(x) \cdot y_{i+1}\right|=\left|\left(A_{i}^{*}-A_{i}(x)\right) \cdot\left(y_{i}-y_{i+1}\right)\right| \\
& =\left|\frac{\frac{2}{3}-2 A_{i}(x)+\frac{4}{3} A_{i}{ }^{2}(x)-A_{i}{ }^{3}(x)}{2-A_{i+1}(x) A_{i}(x)} \cdot\left(y_{i}-y_{i+1}\right)\right| \leq \frac{1}{2}\left|y_{i}-y_{i+1}\right| \leq \frac{1}{2} \mid s^{\prime} \|_{\infty} h
\end{aligned}
$$

We can have $\left\|s-\overline{S_{1}}\right\|_{\infty} \leq\left\|s-F_{1}\right\|_{\infty}+\left\|\overline{S_{1}}-F_{1}\right\|_{\infty} \leq \frac{1}{8}\left\|s s^{\prime \prime}\right\|_{\infty} h^{2}+\frac{1}{2}\left\|s^{\prime}\right\|_{\infty} h$.

Similarly, $\left\|\overline{S_{2}}-s\right\|_{\infty} \leq \frac{1}{8}\left\|s^{\prime \prime}\right\|_{\infty} h^{2}+\frac{1}{3}\left\|s^{\prime}\right\|_{\infty} h$.

Remark 3.1 In the former derivation of $\bar{s}^{(x)}$, we suppose $s(x)$ is a monotone function. But to non-monotonic function, we can divide $X$ into several small intervals to make sure $s(x)$ monotonous in each interval. If $\bar{s}(x)$ can approximate to $s(x)$ in each interval, then $\bar{s}(x)$ can approximate to $s(x)$ in the whole universe.

Remark 3.2 For any given error, we can figure out the number of fuzzy inference rules based on theorem 3.4. In other words, the fuzzy systems based on the center of gravity defuzzifier and triple I algorithm can approximate original system with the desired accuracy.

Example Consider the nonlinear system:

$$
s(x)=\frac{\sin x}{x}, x \in[-3,3] .
$$

Then, We can calculate out that $\left\|s^{\prime \prime}\right\|_{\infty}=0.33,\left\|s^{\prime}\right\|_{\infty}=0.44$.

(1) We use the fuzzy system (16) to approximate the system $s(x)$ within a given level of accuracy.

1) Let $\varepsilon=0.1$, the number of fuzzy inference rules $n=16$ can be obtained by $\frac{1}{8}\left\|s^{\prime \prime}\right\| h^{2}+\frac{1}{2}\left\|s^{\prime}\right\|_{\infty} h<0.1$. The simulate curve and the error curve are shown in fig. 1.

2) Let $\varepsilon=0.05$, the number of fuzzy inference rules $n=28$ can be obtained by $\frac{1}{8}\left\|s^{\prime \prime}\right\| h^{2}+\frac{1}{2}\left\|s^{\prime}\right\|_{\infty} h<0.05$. The simulate curve and the error curve are shown in fig. 2 .
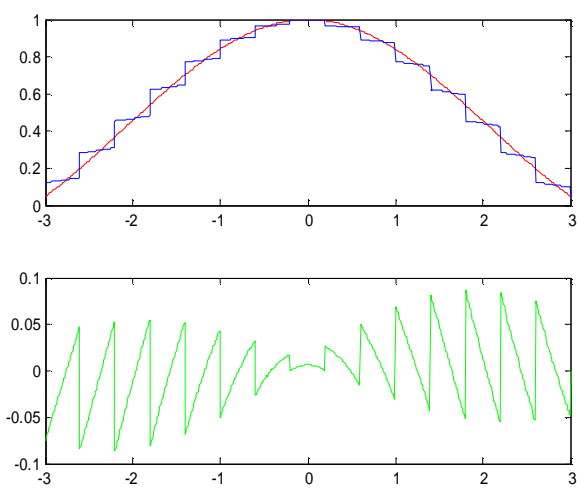

Fig.1
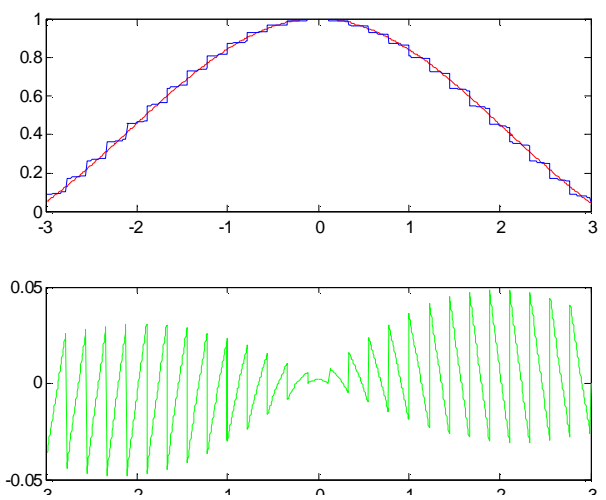

Fig.2 
(2) We use the fuzzy system (17) to approximate the system $s(x)$ within a given level of accuracy.

1) Let $\varepsilon=0.1$, the number of fuzzy inference rules $n=12$ can be obtained by $\frac{1}{8}\left\|s^{\prime \prime}\right\| h^{2}+\frac{1}{3}\left\|s^{\prime}\right\|_{\infty} h<0.1$.

2) Let $\varepsilon=0.1$, the number of fuzzy inference rules $n=20$ can be obtained by $\frac{1}{8}\left\|s^{\prime \prime}\right\| h^{2}+\frac{1}{3}\left\|s^{\prime}\right\|_{\infty} h<0.05$.

The simulate curve and the error curve are omitted.

\section{Conclusions}

Based on a set of input - output data and triple I algorithm, we have built the joint probability density functions of certain two-dimensional random variable respectively by utilizing the singleton fuzzifier and the non-singleton fuzzifier. And we deduced its marginal probability density functions and numerical characteristics. Then we have gotten the generalized center of gravity fuzzy systems and the center of gravity fuzzy systems and we have pointed out that the center of gravity fuzzy systems were regression functions in the sense of probability theory and had the first-order approximation accuracy.

Here, we should point out that in data processing of fuzzy systems, we usually adopt singleton fuzzifier since the input - output data are mostly distinct. However, when the data contains interfering signals, we must consider this uncertain factor. In order to improve anti-interference ability and robustness of the fuzzy system, we should adopt non-singleton fuzzy system. And it has a high application value in the practical projects for its strong pre-filtering capability ${ }^{[12]}$.

\section{References}

[1] Zadeh L A. Fuzzy sets [J]. Information and Control. 1965.8:338 353.

[2] Zadeh L A. Outline of a new approach to the analysis of complex systems and decision processes [J]. IEEE Transactions on Systems. Man, and Cybernetics, 1973,3:28 44.

[3] Zadeh L A. Quantitative fuzzy semantics [J]. Information Sciences, 1971,3:159 176.

[4] Lixin Wang, Fuzzy System and Fuzzy Control tutorial, Tsinghua UniversityPress, (2003)1-99.

[5] Hongxing Li, Fei You, Jiayin Peng, Wenyi Zeng. "Based on fuzzy implication operator of fuzzy controller and its response function". Progress in Natural Science, 2003; 13 (10) :1073-1077.

[6] Guojun Wang. "Fuzzy reasoning algorithm for the whole implication 3 I". Science, E Series, 1999; 29 (1) :32-42.

[7] Jian Hou, Fei You, and Hongxing Li. The modeling and the response function of the fuzzy controller based on the triple I algorithm. Progress in Natural Science. 2005.15（1） : 29

[8] Hongxing Li. "Interpolation mechanism of fuzzy control”. Science, E Series, 1998; 28 (3): 259-267.

[9] Hongxing Li. "The probability of fuzzy systems". Science, E Series, 2006; 36 (4) :373-397.

[10] Xuehai Yuan, Hongxing Li. "Based on the probability distribution of input and output data and digital features". Fuzzy Systems and Mathematics. (unpublished)

[11] Hongxing Li, Jiayin Peng, JiaYin Wang. "Common fuzzy implication operator of fuzzy systems and their response functions". Control Theory and Applications, 2005; 22 (3) :341-347.

[12]Jiakui Zu, Guanzhong Dai, Jun Ma. The non-singleton fuzzy logic system with the pre-filtering characteristics. Control and decision. 2002;17(4). 
